\title{
Author Correction: Large-scale generation of functional mRNA-encapsulating exosomes via cellular nanoporation
}

Zhaogang Yang, Junfeng Shi, Jing Xie, Yifan Wang (D), Jingyao Sun, Tongzheng Liu, Yarong Zhao, Xiuting Zhao, Xinmei Wang, Yifan Ma, Veysi Malkoc, Chiling Chiang, Weiye Deng (D), Yuanxin Chen, Yuan Fu, Kwang J. Kwak, Yamin Fan, Chen Kang, Changcheng Yin, June Rhee, Paul Bertani, Jose Otero, Wu Lu, Kyuson Yun, Andrew S. Lee, Wen Jiang (D, Lesheng Teng (iD, Betty Y. S. Kim (1) and L. James Lee (D)

Correction to: Nature Biomedical Engineering https://doi.org/10.1038/s41551-019-0485-1, published online 16 December 2019.

In the version of this Article originally published, the following images were incorrect owing to the wrong representative images being selected from among images that had been acquired, processed and stored on the in vivo imaging system used in this work: Fig. $7 \mathrm{a}$, top row, labelled 'PBS', and bottom row, labelled 'Exo-T'; Fig. 8e, labelled 'Exosome'; and Fig. 8g, all images in row 5 ('Day 3') and row 6 ('Day 0'). The original and corrected versions of Figs. 7a, $8 \mathrm{e}$ and $8 \mathrm{~g}$ are shown below. In addition, in the Methods section 'In vivo imaging', the following sentence has been amended to clarify that both luminance and fluorescence were recorded by the IVIS Spectrum in vivo imaging system: "At $1 \mathrm{~h}$ and $4 \mathrm{~h}$ after injection, the mice were anaesthetised with $10 \%$ chloral hydrate and recorded by IVIS Spectrum (PerkinElmer)." has been changed to "At $1 \mathrm{~h}$ and $4 \mathrm{~h}$ after injection, the mice were anaesthetized with $10 \%$ chloral hydrate. Luminance and fluorescence were then recorded by an IVIS Spectrum in vivo imaging system (PerkinElmer)." Furthermore, we wish to make clear that for the 'Exosome' group shown in the bottom row in Fig. 8a, the luminescence and fluorescence images labelled 'Exosome' and ' $1 \mathrm{~h}$ ' were taken for the same mouse $1 \mathrm{~h}$ after injection, thus the following sentence has been added to Fig. 8 caption: "In a, the luminescence and fluorescence images for the exosome group labelled 'Exosome' and ' 1 h' correspond to the same mouse." All versions of the Article have been corrected.
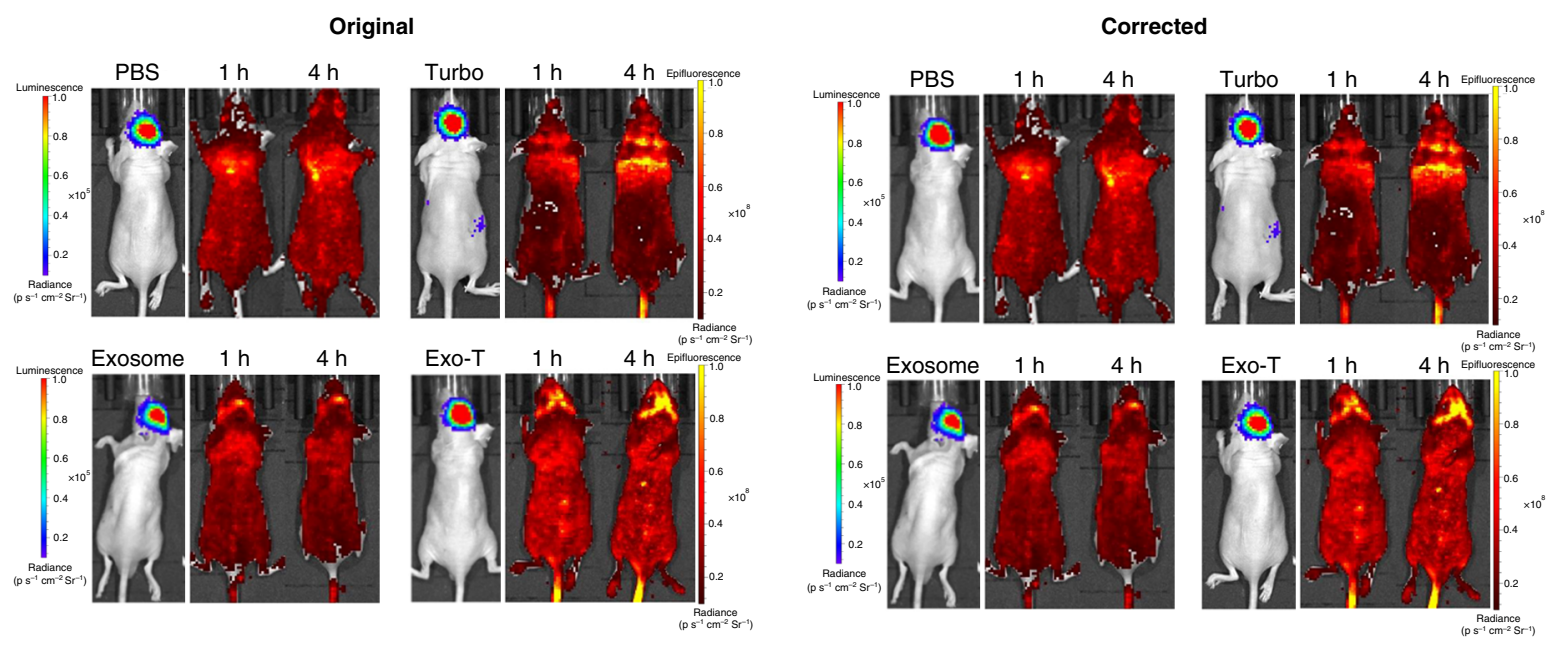

Fig. 7a | Original and Corrected. 


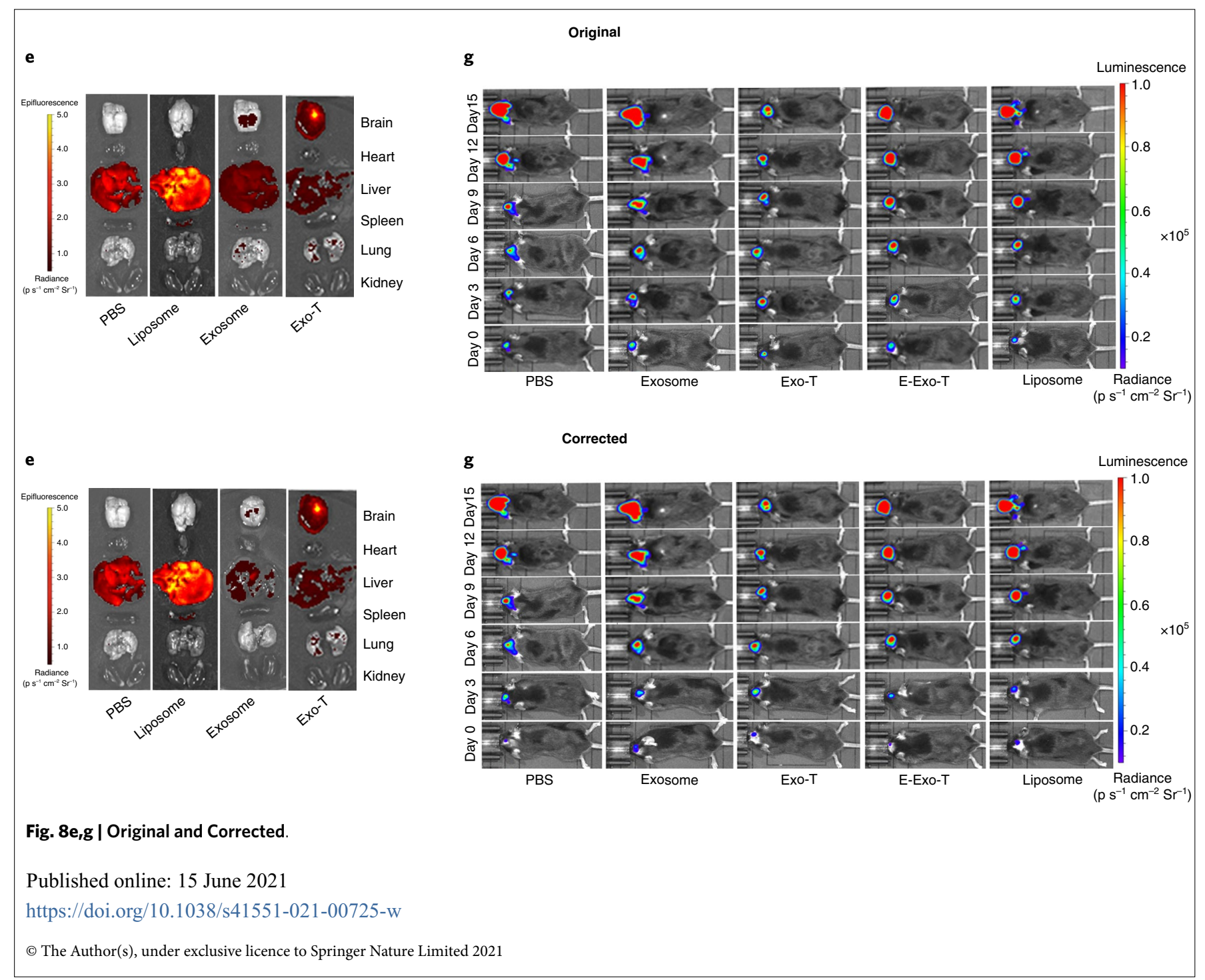

